\title{
Innate immunity to carcinomas?
}

Groh V, Rhinehart R, Secrist H, Bauer S, Grabstein $\mathrm{KH}$, Spies T. Broad tumor-associated expression and recognition by tumor-derived $\gamma \delta$ T cells of MICA and MICB. Proc Natl Acad Sci USA 1999;96:6879-84.

\begin{abstract}
Human MHC class I-related molecules, MICA and MICB, are stress-induced antigens that are recognized by a subset of gamma delta $T$ cells expressing the variable region Vdelta1. This functional association has been found to be limited to intestinal epithelium, where these $T$ cells are prevalent and where MICA and, presumably, MICB are mainly expressed. However, increased frequencies of Vdelta1 gamma delta $T$ cells have been observed in various epithelial tumors; moreover, MICA/B are expressed on diverse cultured epithelial tumor cells. With freshly isolated tumor specimens, expression of $\mathrm{MICA} / \mathrm{B}$ was documented in many, but not all, carcinomas of the lung, breast, kidney, ovary, prostate, and colon. In tumors that were positive for $\mathrm{MICA} / \mathrm{B}$, the frequencies of Vdelta1 gamma delta $T$ cells were significantly higher than in those that were negative. Vdelta1 gamma delta $T$ cell lines and clones derived from different tumors recognized MICA/B on autologous and heterologous tumor cells. In accord with previous evidence, no constraints were observed in these interactions, such as those imposed by specific peptide ligands. Thus, MICA/B are tumor-associated antigens that can be recognized, in an apparently unconditional manner, by a subset of tumorinfiltrating gamma delta $T$ cells. These results raise the possibility that an induced expression of MICA/B, by conditions that may be related to tumor homeostasis and growth, could play a role in immune responses against tumors.
\end{abstract}

\section{Comment}

A receptor ligand system that can facilitate the unconditional killing of epithelial tumour cells by a subset of tumour infiltrating lymphocytes has been reported by a group at the Fred Hutchinson Cancer Research Centre, Seattle. ${ }^{1}$

We have known for many years that a subset of $\gamma / \delta$ T cells is specifically associated with immune responses at epithelial surfaces. Whereas $\gamma / \delta \mathrm{T}$ cells in the blood predominantly use the $\mathrm{V} \delta 2$ delta chain, recognising soluble non-peptide antigens, the intraepithelial $\gamma / \delta$ T cells use the alternative $\mathrm{V} \delta 1$. Until quite recently there was no known ligand for these cells. Earlier work by this group, which has previously been highlighted in $G u t,^{23}$ demonstrated that these V $\delta 1$ bearing cells recognise the ligands MICA and the closely related MICB, molecules which are distantly related to MHC class I but do not present intracellular peptide antigens. MICA is expressed patchily in normal gut epithelium, possibly induced in response to cellular distress, but not by other normal epithelia. It was therefore hypothesised that $\gamma / \delta \mathrm{T}$ cells using V $\delta 1$ patrol the gut, lysing those epithelial cells expressing $\mathrm{MICA} / \mathrm{B}$ as a consequence of antigenic or chemical assault from the lumen.

When the group analysed a panel of tumours for expression of MICA they found, perhaps surprisingly, that not only was it expressed by most colonic carcinomas but also by the majority of cases of ovarian and renal cell carcinoma and some cases of prostatic, lung, and breast carcinoma. ${ }^{1}$ As before, they found no expression in normal epithelium outside the gut, including in paired samples from tumour resections. Expression of MICA by the tumour cells was also associated with a significantly higher frequency of $\gamma / \delta$ $\mathrm{T}$ cells within the tumour infiltrating lymphocyte populations and, of these, increased proportions came from the $\mathrm{V} \delta 1+$ subset. $\mathrm{V} \delta 1+$ cells are known to expand well in culture and have previously been shown to lyse target cells which express MICA/B. ${ }^{2}$ In this paper the authors demonstrate that $\mathrm{V} \delta 1+$ cells could lyse tumour cells from different sites and different individuals, with no restricting elements, provided the tumour cells express MICA.

It appears however that $\mathrm{V} \delta 1$ is not the only receptor for MICA. Two papers which appeared in Science in July $1999^{4}{ }^{5}$ identified an activating receptor complex NKG2DDAP10 that is expressed by most $\gamma / \delta \mathrm{T}$ cells, CD8+ $\alpha / \beta \mathrm{T}$ cells, and NK cells. These NKG2D expressing $\gamma / \delta$ and NK cells, but not the CD8+ $\alpha / \beta \mathrm{T}$ cells, were shown to have cytolytic activity against transfectants and tumour cell lines expressing MICA. This activity could be abolished by addition of monoclonal antibody to NKG2D. The relationship between the NKG2D receptor and V $\delta 1$ receptor is unclear, as the cytolytic activity could also be inhibited by a monoclonal antibody to the $\gamma / \delta$ TCR. $^{2}$ The authors suggest that for the most efficient lysis of MICA+ target cells by $\gamma / \delta$ cells, engagement of both receptors may be necessary. ${ }^{6}$

What practical significance does this work have? It is not yet known what importance the MICA system has for in vivo control of tumours. The frequency of $\gamma / \delta \mathrm{T}$ cells within epithelial tumours is very low, ${ }^{17}$ and so can they really make a significant contribution to the antitumour response? There is certainly no sign of a running battle in vivo; tumour infiltrating $\gamma / \delta \mathrm{T}$ cells are not commonly surrounded by cellular debris of lysed target cells. Tumour cells are known to have many mechanisms allowing them to evade or suppress the immune response. ${ }^{8}$ Perhaps there are factors in vivo which restrict expression of the cytolytic response through MICA, so that the full potential of the antitumour activity mediated by this receptor ligand system can only be observed in vitro. Of course the number of tumours which might be prevented by the activity of the MICA binding sentinels is not quantifiable, and it may be that when tumour growth is observed, we are witnessing a rare failure of the system. Someday it may be possible to enhance the cytolytic response in vivo, or to boost the numbers of $\gamma / \delta$ and NK cells reaching the tumour sites, 
allowing the patient's own cells to destroy the tumour. For now however, it remains to be seen whether the MICA/NKG2D/DAP10 system will prove to be a useful weapon in the war against epithelial tumours and their metastases.

$$
\begin{array}{r}
\text { S J C GOLBY } \\
\text { J SPENCER } \\
\text { Department of Histopathology, } \\
\text { Guy's Kings and St Thomas'Medical School, } \\
\text { London, UK }
\end{array}
$$

Correspondence to: Dr J Spencer, Department of Histopathology, GKT

Medical School, St Thomas' Campus, Lambeth Palace Rd, London SE1 7EH, UK.

Email: jo.spencer@kcl.ac.uk
1 Groh V, Rhinehart R, Secrist H, et al. Broad tumor-associated expression and recognition by tumor-derived $\gamma \delta$ T cells of MICA and MICB. Proc Natl and recognition by tumor-derived

2 Groh V, Steinle A, Bauer S, et al. Recognition of stress-induced MHC molecules by intestinal epithelial $\gamma \delta$ T cells. Science 1998;279:1737-40.

3 Griffith E, Ramsburg E, Hayday A. Recognition by human gut $\gamma \delta$ cells of stress inducible major histocompatibility molecules on enterocytes. Gut 1998; 43:166-7

4 Bauer S, Groh V, Wu J, et al. Activation of NK cells and T cells by NKG2D, a receptor for stress inducible MICA. Science 1999;285:727-9.

5 Wu J, Song Y, Bakker ABH, et al. An activating immunoreceptor complex Wu J, Song Y, Bakker ABH, et al. An activating immunor
formed by NKG2D and DAP10. Science 1999;285:730-2

6 Hagmann M. A trigger of natural (and other) killers. Science 1999;285:645-7. 7 Watanabe $\mathrm{N}$, Hizuta A, Tanaka $\mathrm{N}$, et al. Localisation of $\mathrm{T}$ cell receptor (TCR) $-\gamma \delta+\mathrm{T}$ cells into human colorectal cancer: flow cytometric analysis of TCR- $\gamma \delta$ expression in tumour-infiltrating lymphocytes. Clin Exp Immunol 1995;102:167-73.

8 Finke J, Ferrone S, Frey A, et al. Where have all the T cells gone. Immunol Today 1999;20:158-60. 